\title{
Investigação radiométrica da Praia de Meaípe em Guarapari-ES
}

\author{
Calheiro, D.S. ${ }^{1}$, Passamai, Jr.J.L. ${ }^{2 *}$ \\ 1 Pós-Graduação em Física de Materiais (FIMAT), UFOP, Ouro Preto, MG, Brasil. \\ 2 Departamento de Física, Universidade Federal do Espírito Santo, Vitória, ES, Brasil.
}

*e-mail: passamaijr@gmail.com

\section{Resumo}

O objetivo do nosso trabalho foi investigar a atividade radioativa na Praia de Meaípe na cidade de Guarapari-ES utilizando uma metodologia dinâmica (variação com tempo). As medidas foram tomadas em tempos distintos durante o período de (março a agosto de 2016). Para tanto um ciclocomputador Etrex-10 foi usado para determinar uma rota na areia da praia usada por banhistas e também um contador Geiger que mediu e armazenou os dados. Foi possível observar que existem alguns fatores que podem contribuir para a variação do nível da radiação como: maré, clima e estação do ano.

\begin{abstract}
The objective of our work was to investigate the radioactive activity in Praia de Meaípe in the city of Guarapari-ES using a dynamic methodology (variation with time). Measures were taken at different times during the period from March to August 2016. An Etrex-10 cyclocomputer was used to determine a route in the beach sand used by bathers and also a Geiger counter that measured and stored the data. It was possible to observe that there are some factors that can contribute to the variation of the radiation level as: tide, climate and season of the year.
\end{abstract}

\section{Introdução}

$\mathrm{O}$ relato de Orlando et al. [1] indica uma possível relação da atividade radioativa nas praias de Guarapari com efeitos biológicos. Indiferente ao efeito da radiatividade, verificase que em geral os autores, anteriores a este estudo, descrevem as propriedades das areias utilizando amostras retiradas em um único local, de forma singular no tempo $[2,3,4]$. Esses trabalhos tiram correlações sobre uma amostra singular e com uma composição não reprodutível no tempo.Neste trabalho foi realizada a medição do nível da atividade radioativa $(\mu \mathrm{Sv} / \mathrm{h})$ presente na praia de Meaípe variando no tempo, os estudos começaram em março de 2016 e foram até agosto de 2016. Nesses seis meses de medidas, o intervalo entre as medidas foi de 7 dias a 15 dias, sendo feita apenas duas medidas por mês.
Para a rota ser seguida várias vezes no mesmo caminho utilizamos as coordenadas de georreferenciamento da tabela a seguir. 
Tabela 1: Coordenadas da rota utilizada.

\begin{tabular}{|c|c|c|c|}
\hline $20^{\circ} 44^{\prime} 22.70^{\prime \prime} \mathrm{S}$ & $040^{\circ} 32^{\prime} 10.79^{\prime \prime} \mathrm{W}$ & $20^{\circ} 44^{\prime} 20.82^{\prime \prime} \mathrm{S}$ & $040^{\circ} 32^{\prime} 43.55^{\prime \prime} \mathrm{W}$ \\
\hline $20^{\circ} 44^{\prime} 21.55^{\prime \prime S}$ & $040^{\circ} 32^{\prime} 12.10^{\prime \prime} \mathrm{W}$ & $20^{\circ} 44^{\prime} 21.10^{\prime \prime S}$ & $040^{\circ} 32^{\prime} 45.25^{\prime \prime} \mathrm{W}$ \\
\hline $20^{\circ} 44^{\prime} 20.63^{\prime \prime S}$ & $040^{\circ} 32^{\prime} 13.52^{\prime \prime} \mathrm{W}$ & $20^{\circ} 44^{\prime} 21.20^{\prime \prime} \mathrm{S}$ & $040^{\circ} 32^{\prime} 46.94^{\prime \prime} \mathrm{W}$ \\
\hline $20^{\circ} 44^{\prime} 19.80^{\prime \prime S}$ & $040^{\circ} 32^{\prime} 15.09^{\prime \prime} \mathrm{W}$ & $20^{\circ} 44^{\prime} 21.41^{\prime \prime S}$ & $040^{\circ} 32^{\prime} 48.66^{\prime \prime} \mathrm{W}$ \\
\hline $20^{\circ} 44^{\prime} 19.06^{\prime \prime} \mathrm{S}$ & $040^{\circ} 32^{\prime} 16.64 " \mathrm{~W}$ & $20^{\circ} 44^{\prime} 21.80^{\prime \prime} \mathrm{S}$ & $040^{\circ} 32^{\prime} 50.32^{\prime \prime} \mathrm{W}$ \\
\hline $20^{\circ} 44^{\prime} 18.52 " \mathrm{~S}$ & $040^{\circ} 32^{\prime} 18.29^{\prime \prime} \mathrm{W}$ & $20^{\circ} 44^{\prime} 22.39^{\prime \prime} \mathrm{S}$ & $040^{\circ} 32^{\prime} 51.93^{\prime \prime} \mathrm{W}$ \\
\hline $20^{\circ} 44^{\prime} 18.06^{\prime \prime S}$ & $040^{\circ} 32^{\prime} 19.96^{\prime \prime} \mathrm{W}$ & $20^{\circ} 44^{\prime} 23.16^{\prime \prime S}$ & $040^{\circ} 32^{\prime} 53.42^{\prime \prime} \mathrm{W}$ \\
\hline $20^{\circ} 44^{\prime} 17.61 " \mathrm{~S}$ & $040^{\circ} 32^{\prime} 21.70^{\prime \prime} \mathrm{W}$ & $20^{\circ} 44^{\prime} 24.03 " \mathrm{~S}$ & $040^{\circ} 32^{\prime} 54.84^{\prime \prime} \mathrm{W}$ \\
\hline $20^{\circ} 44^{\prime}$ & $.44 " \mathrm{~W}$ & $4.95 \mathrm{~s}$ & $040^{\circ} 32^{\prime} 56.26^{\prime \prime} \mathrm{W}$ \\
\hline $20^{\circ} 44^{\prime}$ & $040^{\circ}$ & $20^{\circ} 44^{\prime} 25.93 " \mathrm{~S}$ & $040^{\circ} 32^{\prime} 57.61^{\prime \prime} \mathrm{W}$ \\
\hline $20^{\circ} 44^{\prime}$ & $040^{\circ}$ & $.01 " \mathrm{~S}$ & $040^{\circ}$ \\
\hline $20^{\circ} 4$ & $040^{\circ}$ & $.93 " \mathrm{~S}$ & $040^{\circ} 33^{\prime} 00.29^{\prime \prime}$ \\
\hline $20^{\circ} 44^{\prime} 17.68^{\prime \prime S}$ & $040^{\circ} 32^{\prime} 32.03^{\prime \prime} \mathrm{W}$ & $20^{\circ} 44^{\prime} 28.62 " \mathrm{~S}$ & $040^{\circ} 33^{\prime} 01.86^{\prime \prime} \mathrm{W}$ \\
\hline $20^{\circ} 44^{\prime} 17.92^{\prime \prime} \mathrm{S}$ & $040^{\circ} 32^{\prime} 33.72^{\prime \prime} \mathrm{W}$ & $20^{\circ} 44^{\prime} 29.39^{\prime \prime S}$ & $040^{\circ} 33^{\prime} 03.38^{\prime \prime} \mathrm{W}$ \\
\hline $20^{\circ} 44^{\prime} 18.26^{\prime \prime S}$ & $040^{\circ} 32$ & $20^{\circ} 44^{\prime} 30.38^{\prime \prime S}$ & $040^{\circ} 33^{\prime} 04.75^{\prime \prime} \mathrm{W}$ \\
\hline $20^{\circ} 44^{\prime}$ & $040^{\circ} 3$ & $20^{\circ} 44^{\prime} 31.33 " \mathrm{~S}$ & $040^{\circ} 33^{\prime} 06.13^{\prime \prime} \mathrm{W}$ \\
\hline $20^{\circ} 44^{\prime} 19.27^{\prime \prime S}$ & $040^{\circ} 32^{\prime} 38.72^{\prime \prime} \mathrm{W}$ & $20^{\circ} 44^{\prime} 32.29^{\prime \prime S}$ & $040^{\circ} 33^{\prime} 07.51^{\prime \prime} \mathrm{W}$ \\
\hline $20^{\circ} 44^{\prime} 19.76^{\prime \prime S}$ & $040^{\circ} 32^{\prime} 40.32^{\prime \prime} \mathrm{W}$ & $20^{\circ} 44^{\prime} 33.07 " \mathrm{~S}$ & $040^{\circ} 33^{\prime} 08.99^{\prime \prime} \mathrm{W}$ \\
\hline $20^{\circ} 44^{\prime} 20.30$ & $040^{\circ} 32^{\prime} 41.93^{\prime \prime} \mathrm{W}$ & $20^{\circ} 44^{\prime} 33.60^{\prime \prime S}$ & $040^{\circ} 33^{\prime} 10.01^{\prime \prime} \mathrm{W}$ \\
\hline
\end{tabular}

\section{Resultados e Discussões}

$\mathrm{Na}$ figura 01, registramos as três primeiras medidas realizadas entre o mês de março e abril de 2016 e obtivemos o seguinte gráfico.

Fig. 01. Gráfico das três primeiras medidas.

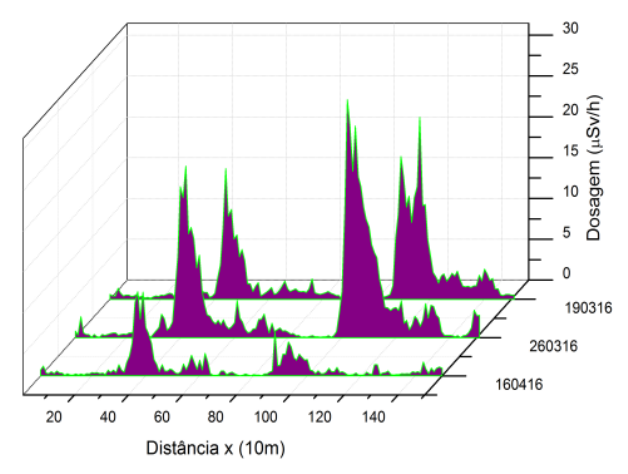

Á direita do gráfico acima temos 3 datas que foram feitas as medidas, a primeira foi feita em 19/03/16 a segunda em 26/03/16 e a terceira em 16/04/16.

Durante a primeira medida verificamos uma dose de aproximadamente 22,2 $(\mu \mathrm{Sv} / \mathrm{h})$ em $1160 \mathrm{~m}$, na segunda medida verificamos uma dose de 29,05 ( $\mu \mathrm{Sv} / \mathrm{h})$ em $1020 \mathrm{~m}$, na terceira medida verificamos uma dosagem 10,28 $(\mu \mathrm{Sv} / \mathrm{h})$ em $370 \mathrm{~m}$.

Realizamos mais medidas e obtivemos 0 gráfico a seguir.

Fig. 02. Gráfico das três últimas medidas.

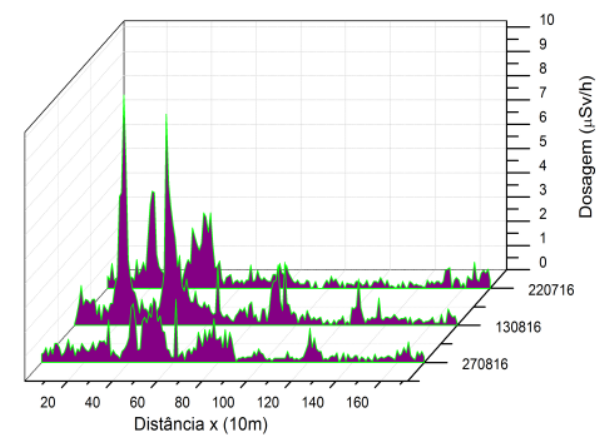

A medida do dia 13/08/16 mostra a dosagem de 4,00 ( $\mu \mathrm{Sv} / \mathrm{h})$ em $210 \mathrm{~m}$, na segunda medida verificamos uma dose de $9,49(\mu \mathrm{Sv} / \mathrm{h}) \mathrm{em}$ $230 \mathrm{~m}$, na terceira medida verificamos uma dosagem $2,65(\mu \mathrm{Sv} / \mathrm{h})$ próximo de $500 \mathrm{~m}$.

\section{Conclusões}

Nossas medições neste trabalho mostram que a radiação nesta praia varia de intensidade com o tempo e se desloca no espaço continuamente.

Por ser uma praia que fica entre em região de alta concentração de radioatividade ela foi causou surpresa por estar com níveis que não chegaram em $0,8(\mu \mathrm{Sv} / \mathrm{h})$.

O ponto a ser ressaltado aqui é de que análises instantâneas de amostras de areia não caracterizam bem a Praia de Meaípe, uma vez que o sistema é dinâmico.

Somente uma análise temporal com base em 1 ano pode fornecer valores médios da atividade radioativa na Praia de Meaípe. 
Guarapari, Espírito Santo State, Brazil. (2013).

\section{Referências}

[1] Orlando, M T D, et al. Correlation between Breast Cancer and Radiation Level of Guarapari City - ES, Blucher Proceedings ISSN: $2358-2359 \quad \mathrm{~V}$ 01, (2014) $\mathrm{n} \quad 02$ DOI:10.5151/phypro-ecfa-020

[2] Fujinami, N.; T. Koga, T.; Morishima. H. External Expousure Rates From Terrestrial Radiantion At Guarapari And Meaipe In Brazil. (1999).

[3] Vasconcelos, D.C, Et Al. Modelling Natural Radioactivity In Sand Beaches Of
[4] Calheiro, D.S.; Passamai. Jr.,J.L. Estudo da Radiação na areia da Praia da Areia Preta. (2016). DOI: $10.5151 /$ phypro-vii-efa-035

Agradecimentos.

Comissão Nacional de Energia Nuclear (CNEN), ao CNPQ e a Fundação de Amparo à Pesquisa e Inovação do Espírito Santo (FAPES) (projetos 098/2019 e 270/2019). 